\title{
Sonolência diurna excessiva em trabalhadores da área de enfermagem
}

Excessive daytime sleepiness in nursing workers

José Carlos Souza'

\section{RESUMO}

Objetivo: A sonolência diurna excessiva (SDE) caracteriza-se por episódios de sono em situações em que o indivíduo deveria estar acordado. Objetivou-se detectar a prevalência de SDE em trabalhadores da área de enfermagem. Métodos: Fez-se um estudo, descritivo e de corte transversal em 226 trabalhadores de enfermagem de um hospital público de Campo Grande, MS. Foi aplicada a Escala de Sonolência de Epworth (ESE). Resultados: Foi encontrada uma prevalência de 30,09\% de SDE. Não foram detectadas relações entre SDE e

\section{Palavras-chave}

Sonolência diurna excessiva, prevalência, enfermagem.

\section{Key-words}

Excessive daytime sleepiness, prevalence, nursing. gênero, idade, trabalho em turnos, uso de hipnóticos; 27,64\% trabalhavam em turnos fixos e 32\% alternados. Conclusões: Foi alta a prevalência de SDE em trabalhadores da área de enfermagem na amostra avaliada. Sugere-se que medidas sejam tomadas para uma abordagem adequada deste transtorno neste grupo.

\begin{abstract}
Objective: Excessive daytime sleepiness (EDS) is characterized by episodes of naps or moments of sleep in situations where the individual should be awake. The objective was to detect the prevalence of EDS in nursing professionals. Methods: A transverse study was carried out, descriptive, in 226 workers in the nursing in a public hospital in Campo Grande, Mato Grosso do Sul. The Epworth Sleepiness Scale (ESS) was applied. Results: It was found a prevalence of 30,09\% of sleepiness. No connection was detected in the occurrence of EDS between the sexes, ages, shift work and use of hypnotics. 27,64\% worked in fixed shift and 32,00\% in alternating shift. Conclusions: The prevalence of EDS manifested among nursing professionals was shown to be high. Steps should be taken to provide treatment for this disturbance.
\end{abstract}

\section{INTRODUÇÃO}

Os distúrbios do sono têm sido reconhecidos como sérios problemas de saúde pública, particularmente em relação à sonolência diurna excessiva (SDE) (Dement e Mitler, 1993). O termo sonolência é empregado aqui como a propensão a cochilar ou a adormecer quando a intenção é ficar acordado, distinguindo-se do cansaço ou fadiga, que não estão sempre relacionados à sonolência (Johns e Hocking, 1997). Existem vários censos epidemiológicos mundiais indicando que a prevalência da SDE, em adultos, na população geral está entre 0,3\% e 13,3\% (D’Alessandro et al., 1995). Entretanto, cada um destes estudos utilizou uma metodologia diferente para medir a sonolência, o que torna difícil

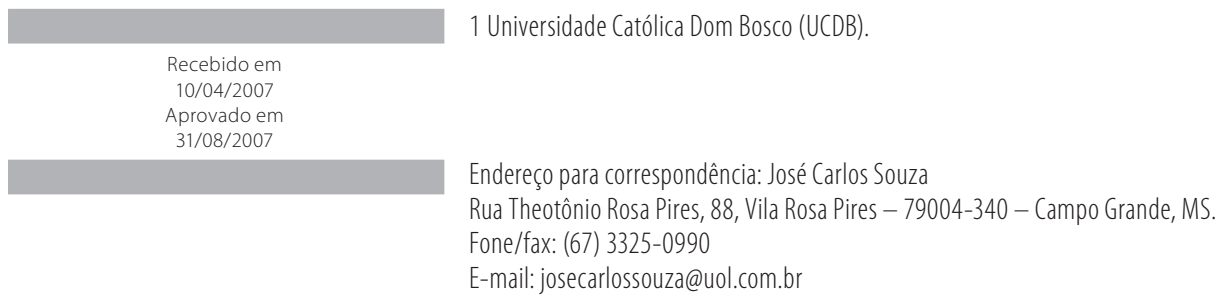


a comparação dos dados. Por isso, a escala de sonolência de Epworth (ESE) foi escolhida para esta pesquisa, pois é um instrumento simples e bastante utilizado em estudos mundiais e pelo grupo "Sono, Sonhos e seus Distúrbios", da Universidade Católica Dom Bosco (UCDB), vinculado ao Conselho Nacional de Desenvolvimento Científico e Tecnológico (CNPq) (Johns, 1991, 1992, 1993, 1994; Souza et al., 2002; Souza et al., 2003). Quanto ao trabalho em turnos alternados (shift-work), a literatura mundial também é extensa (Mazzetti-di-Pietralata et al., 1990; Bobko, 1994; Gupta e Pati, 1994; Campbell, 1995; Srithongchai e Intaranont, 1996; Benoit, 1996; Matsumoto et al., 1996; Fukuda, 1997; Fitzpatrick et al., 1999). O emprego de turnos de trabalho em horas habitualmente dedicadas ao sono é fruto da necessidade de produção e funcionamento contínuo de nossa sociedade. Essas tarefas englobam turnos fixos noturnos ou turnos rotatórios (regulares ou irregulares), geralmente de oito ou 12 horas de duração. Os distúrbios do sono produzidos por estas rotinas de trabalho consistem fundamentalmente nos sintomas de insônia e de SDE. Além dos distúrbios do sono mencionados, os trabalhadores em turnos apresentam maior risco para enfermidades cardiovasculares, gastrintestinais e infertilidade. A sonolência excessiva e o déficit de atenção durante o trabalho, e fora dele, implicam também riscos para a segurança do trabalhador e seu relacionamento. Numerosos acidentes, nos quais o fator humano é considerado responsável, ocorrem em horários de maior tendência a dormir (Fischer et al., 2003).

Entre os trabalhadores da área de enfermagem, o shiftwork interfere em sua performance, sua saúde física, psicológica e ocupacional. Os turnos com intervalo de 12 horas e meia estão associados a menores prejuízos (Fitzpatrick et al., 1999). A necessidade de adaptar sua vida às alternâncias de turno de trabalho gera no trabalhador da área de saúde expectativa e preocupações que, por conseguinte, afetam a sua qualidade de vida (Srithongchai e Intaranont, 1996). O trabalho, assim, por mais prazeroso e interessante que seja, pode se tornar um propulsor de doenças. Estes fatores são sobrepostos, muitas vezes, por necessidades financeiras, até que o próprio organismo manifeste sintomas de alerta ou de exaustão global (Ferreira, 1985; Bonnet, 1986; Manocchia et al., 2001).

O objetivo deste estudo foi detectar a prevalência de SDE em trabalhadores da área de enfermagem.

\section{MÉTODO}

Fez-se um estudo descritivo e de corte transversal. A amostra foi de 226 trabalhadores da área de enfermagem de um hospital público de Campo Grande, MS. Foi aplicada a ESE e um Termo de Consentimento Livre e Esclarecido a ser assinado pelos sujeitos da pesquisa. Houve a homologação da Comissão de Ética e Pesquisa do Hospital das Clínicas da Faculdade de Medicina da Universidade de São Paulo. Entre as mais empregadas, mundialmente, está a ESE, desenvolvida por Murray W. Johns, no Centro de Distúrbios do Sono do Epworth Hospital, Melbourne, Austrália (Johns, 1991). Esta foi planejada como um questionário simples, de fácil aplicação, com o intuito de fornecer uma medida geral do grau de sonolência diurna. Na aplicação da ESE pede-se à pessoa para estimar a tendência para adormecer em oito situações monótonas da vida diária, ressaltando que não se trata de fadiga simplesmente. O entrevistado deve fornecer uma nota de zero a três, quantificando sua tendência (probabilidade) para adormecer; as notas dadas a cada uma das oito questões são somadas; o total de 10 ou mais pontos é indicativo da presença de SDE e 16 ou mais de distúrbio respiratório ou síndrome da apnéia do sono. Os dados foram tabulados e analisados por meio da estatística descritiva e dos testes $t$ de Student, qui-quadrado, Anova e teste de Fisher.

\section{RESULTADOS}

No total de 226 sujeitos, 15 eram enfermeiros (6,64\%), 64 técnicos de enfermagem (28,32\%) e 147 auxiliares de enfermagem (65,04\%).

A tabela 1 mostra que os enfermeiros correspondem ao grupo mais jovem. Ao analisar as médias das pontuações entre as funções encontrou-se $F=1,19$ e valor de $p=0,3060$, indicando não haver diferença entre os grupos quanto à pontuação.

Ao analisar as médias das idades entre os grupos encontrou-se $F=1,72$ e valor de $p=0,1910$, indicando não haver diferença entre os grupos quanto à idade.

Não há diferença entre as funções quanto à ocorrência de SDE. Não há, também, diferença entre os gêneros quanto à ocorrência de SDE $(p=0,2621)$ e entre os grupos que trabalham em turnos e os que não trabalham $(p=0,6490)$.

Tabela 1. Distribuição dos trabalhadores quanto à função, a pontuação na ESE e a idade

\begin{tabular}{|c|c|c|c|c|c|}
\hline \multirow{2}{*}{ Grupo avaliado } & \multirow{2}{*}{ Número de avaliados (\%) } & \multicolumn{2}{|c|}{ Pontuação } & \multicolumn{2}{|c|}{ Idade } \\
\hline & & Média \pm desvio-padrão & Variância & Média \pm desvio-padrão & Variância \\
\hline Enfermeiros & $15(6,64 \%)$ & $9,93 \pm 2,99$ & 8,92 & $28,80 \pm 7,04$ & 49,60 \\
\hline Técnico de enfermagem & $64(28,32 \%)$ & $8,44 \pm 4,08$ & 16,63 & $34,45 \pm 9,17$ & 84,06 \\
\hline Auxiliar de enfermagem & $147(65,04 \%)$ & $8,34 \pm 3,76$ & 14,16 & $34,09 \pm 8,18$ & 66,87 \\
\hline
\end{tabular}

Ponto de corte utilizado = 10 pontos; sonolência para pontuação superior a 10; normal para pontuação geral igual ou inferior a 10 
A tabela 2 mostra que não há diferença entre os grupos quanto aos turnos serem alternados ou fixos quanto à ocorrência de SDE. O item ignorado refere-se às questões não respondidas quanto ao tipo de turnos.

Não há diferença, também, entre os grupos que tomam ou não alguma medicação para auxiliá-los a dormir quanto à ocorrência de SDE $(p=0,0981)$.

\section{DISCUSSÃO}

A pesquisa realizada evidenciou a existência de SDE em 30,09\% da população entrevistada. Canani e Barreto (2001) destacam que a SDE é uma queixa comum na população geral com prevalência entre 0,5\% e 14\%.

Rodrigues et al. (2002) ressaltam que a SDE afeta principalmente profissionais que prestam cuidados a pacientes crônicos, isso envolve médicos, residentes e profissionais de enfermagem. Estes autores constataram, em uma pesquisa com estudantes de medicina, que $61,62 \%$ deles apresentavam sonolência em algum momento durante o semestre. A SDE pode ser ocasionada principalmente por hábitos de sono inadequados incluindo a sua privação. Os efeitos do acúmulo de sono por uma semana, com duração de 4,5 horas por dia em média, equiparam os níveis de sonolência aos da privação total do sono tornando maior a possibilidade de sono no trabalho (Canani e Barreto, 2001; Fischer et al., 2002).

Referente à relação entre gêneros e a ocorrência de SDE, nesta pesquisa, os dados encontrados mostraram não haver diferenças entre eles. Doi e Minowa (2003) encontraram os mesmos resultados ao realizarem uma pesquisa para detectar relações entre gênero, prevalência e risco para SDE em funcionários de uma empresa de telecomunicações de Tóquio. Os dados obtidos mostraram a prevalência da sonolência em 13,3\% das mulheres e 7,2\% dos homens. Com isso constataram não haver diferenças entre fatores de risco e prevalência da SDE em ambos os gêneros.

Delafosse et al. (2000) comprovaram que algumas enfermeiras não ajustam seu ritmo circadiano ao trabalho noturno. Os autores realizaram uma pesquisa com 15 enfermeiras do turno noturno durante períodos de trabalho e descanso. Verificaram que sete delas apresentavam pelo menos cinco horas de atraso no ciclo sono-vigília durante os períodos de trabalho comparado com os de descanso. Quanto à relação entre idade e sonolência a pesquisa verificou que não há diferença entre idade e o grupo de pessoas que possui SDE e os que não possuem. Matsumoto et al. (1996) destacam que os problemas ligados ao sono e à fadiga aumentam com a idade, mas a percepção subjetiva do sono é maior em indivíduos mais jovens. Conforme Souza et al. (2003), há uma diminuição das horas de sono durante o dia com o aumento da idade, sendo assim, há também maior ocorrência de despertares noturnos e uso de medicação hipnótica. Em relação ao uso de medicamentos para dormir, esta pesquisa detectou não haver diferenças entre os sujeitos que usam ou não esta medicação e a ocorrência de SDE. Porém, o uso de medicamentos e outros meios para ficar acordado encontrado foram maior entre os profissionais que possuem SDE. Em sua pesquisa com enfermeiros que trabalham em dois turnos consecutivos, ou em dois turnos noturnos consecutivos, depois do trabalho diurno, Matsumoto et al. (1996) verificaram que após o primeiro turno noturno de trabalho, os problemas do sono aumentavam. Com isso a freqüência do uso de medicamentos de indução do sono também era maior. Novak e Auvil-Novak (1996) em um estudo com 45 enfermeiros que trabalhavam em turnos de 12 horas constataram que o uso da cafeína (estimulante do sistema nervoso central) era uma prática comum para melhorar o desempenho no trabalho, principalmente no turno noturno.

Os trabalhadores da área de enfermagem do hospital público estudado, neste estudo, tinham à época, características peculiares que poderiam explicar a ausência de diferenças entre a pontuação de sonolência entre os grupos que trabalhavam ou não em turnos, como a existência de folgas espaçadas, predeterminadas, o que facilitava o descanso e a recuperação física destes trabalhadores.

\section{CONCLUSÕES}

É alta a prevalência de SDE entre os trabalhadores da área de enfermagem, aqui estudados, assim como do trabalho em turnos. O fato de as faixas etárias mais jovens serem as mais atingidas pela SDE retrata a necessidade de novos estudos epidemiológicos com esta população e a instituição de programas preventivos.

Tabela 2. Distribuição dos trabalhadores quanto à presença de SDE e o tipo de turnos

\begin{tabular}{|c|c|c|c|c|c|c|}
\hline \multirow{2}{*}{ Diagnóstico } & \multicolumn{2}{|c|}{ Turno alternado } & \multicolumn{2}{|c|}{ Turno fixo } & \multicolumn{2}{|c|}{ Turno ignorado } \\
\hline & $\mathrm{N}^{\circ}$ de avaliados & Porcentagem & $\mathrm{N}^{\circ}$ de avaliados & Porcentagem & № de avaliados & Porcentagem \\
\hline Normal & 68 & 68,00 & 89 & 72,36 & 1 & 33,33 \\
\hline Sonolência & 32 & 32,00 & 34 & 27,64 & 2 & 66,67 \\
\hline Total & 100 & 44,25 & 123 & 54,42 & 3 & 1,33 \\
\hline
\end{tabular}

Qui-quadrado $=0,50$ (por Mantel-Haenszel); valor de $p=0,4793$. 


\section{AGRADECIMENTOS}

Ao Conselho Regional de Enfermagem do Estado de Mato Grosso do Sul (Coren-MS), à diretoria do hospital onde se realizou a pesquisa, aos sujeitos entrevistados e aos alunos da UCDB Neomar Souza, Adelzira Sousa Soares, Juraci Nantes de Almeida, Ana Flávia W. G. Serpa, Luiza Alvarenga Marques, Eliny Salazar e Genilson Romeiro Serpa pelo auxílio na coleta de dados.

\section{REFERÊNCIAS}

Benoit 0. Sleep-wakefulness rhythm disorders. Rev Prat, 46(20): 2442-7, 1996.

Bobko NA. Some biorhythm characteristics of personal adjustment of operators working in a shift regime. Fiziol Zh, 40(2): 31-7, 1994.

Bonnet MH. Performance and sleepiness as a function of frequency and placement of sleep disruption. Psychophysiology, 23(3): 263-71, 1986.

Campbell SS. Effects of timed bright-light exposure on shift-work adaptation in middle-aged subjects. Sleep, 18(6): 408-16, 1995.

Canani SF, Barreto SSM. Sonolência e acidentes automobilísticos. Jornal de Pneumologia, 27(2): 94-6, 2001

D’Alessandro R, Rinaldi R, Cristina E, Gamberini G, Lugaresi E. Prevalence of excessive daytime sleepiness. An open epidemiological problem. Sleep, 18: 389-91, 1995.

Delafosse JY, Léger D, Quera-Salva MA, Samson 0, Adriean J. Comparative study of actigraphy and ambulatory polysomnography in the assessment of adaptation to night shift worke in nurses. Rev Neurol, 156 (6-7): 641-5, 2000

Dement WC, Mitler MM. It's time to wake up to the importance of sleep disorders. JAMA, 269: 1548-9, 1993.

Doi Y, Minowa M. Gender difference in excessive daytime sleepiness among japoneses workeers. Soc Sci Med, 56(4): 883-94, 2003

Ferreira LL. Sono de trabalhadores em turnos alternantes. Rev Bras de Saúde Ocupacional, 51(13): 25-7, 1985

Fischer FM, Teixeira LR, Borges FNS, Gonçalves L, Ferreira RM. Percepção de sono: duração e alerta em profissionais da área de enfermagem. Cadernos de Saúde Pública, 18(5): 1261-9, 2002.
Fischer FM, Castro CR, Rotenberg L. Trabalho em turnos e noturno na sociedade 24 horas. São Paulo: Atheneu, 2003.

Fitzpatrick JM, While AE, Roberts JD. Shift work and its impact upon nurse performance: current knowledge and research issues. J Adv Nurs, 29(1): 18-27, 1999.

Fukuda K. Twins under shift work: a case study of sleep log data. Percept Mot Skills, 84(3Pt1): 931-7, 1997.

Gupta S, Pati AK. Desynchronization of circadian rhythms in a group of shift working nurses: effects of pattern of shift rotation. J Hum Ergol Tokyo, 23(2): 121-31, 1994.

Johns M, Hocking B. Daytime sleepiness and sleep habits of australian workers. Sleep, 20(10): 844-9, 1997.

Johns MW. A new method for measuring daytime sleepiness: the Epworth Sleepiness Scale. Sleep, 14(6): 540-5, 1991

Johns MW. Reliability and factor analysis of the Epworth Sleepiness Scale. Sleep, 15(4): 376-81, 1992.

Johns MW. Daytime sleepiness, snoring and obstructive sleep apnea: the Epworth Sleepiness Scale. Chest, 103: 30-6, 1993.

Johns MW. Sleepiness in different situations measured by the Epworth Sleepiness Scale. Sleep, 17(8): 703-10, 1994

Manocchia M, Keller S, Ware JE. Sleep problems, health-related quality of life, work functioning and health care utilization among the chronically ill. Qual Life Res, 10(4): 331-45, 2001.

Matsumoto M, Kamata S, Naoe H, Mutoh F, Chiba S. Investigation of the actual conditions of hospital nurses working on three rotating shifts: questionnaire results of shift work schedules, feelings of sleep and fatigue, and depression. Seichin Shinkeigaku Zasshi, 98(1): 11-26, 1996

Mazzetti-di-Pietralata M, Ballarati G, Castracane RE, Galanti A, Gallo A, Leonardi C, Mazzetti-diPietralata G, Mensa A, Zulli L. Digestive disturbances in shift-workers: a clinical statistical investigation. Prog Clin Biol Res, 341B: 369-77, 1990.

Novak RD, Auvil-Novak SE. Focus group evaluation of night nurse shiftworke difficulties and coping strategies. Chonobiol Int, 13(6): 457-63, 1996.

Rodrigues RND, Viegas CAA, Silva AA, Tavares P. Daytime sleepiness and academic performance in medical students. Arq Neuropsiquiatr, 60(1): 6-11, 2002.

Souza JC, Reimão R, Magna LA. Excessive daytime sleepiness in campo grande general population, Brazil. Arq Neuropsiquiatr, 60(3): 558-62, 2002.

Souza JC, Magna LA, Paula TH. Sonolência excessiva diurna e uso de hipnóticos em idosos. Rev Psiquiatria Clínica, 30(3): 80-5, 2003

Srithongchai S, Intaranont K. A study of impact of shift work on fatigue level of workers in a sanitary-ware factory using a fuzzy set model. J Hum Ergol Tokyo, . 1996;25(1): 93-9, 1996. 